\title{
PositivelyPregnant: Using guided prompts in a stress-management app for pregnant women
}

\author{
Carrie Barber, Sukrita Singh \\ School of Psychology \\ University of Waikato, New Zealand \\ carrie.barber@waikato.ac.nz,bsukrita@yahoo.in
}

\author{
Annika Hinze, Nicholas Vanderschantz \\ Computer Science Department \\ University of Waikato, New Zealand \\ \{hinze,vtwoz\}@waikato.ac.nz
}

\begin{abstract}
This paper reports on our interdisciplinary research on stress management for pregnant women. We developed a mobile app - PositivelyPregnant - for supporting the mental wellbeing of pregnant women in New Zealand. The app aims to support women in using the time during pregnancy to build resilience and plan a healthy future for the whole family. We report the results from a study using PositivelyPregnant, carried out at 24 weeks gestation with 88 women. We particularly explored the use of prompts for continued engagement.
\end{abstract}

Mobile app, Pregnancy, Perinatal support, Mental health, User study, e-health, Prompts.

\section{INTRODUCTION}

Pregnant women may experience symptoms of anxiety, stress and depression during their pregnancy (Sprenger, Mettler \& Osma, 2017). Maternal stress during pregnancy has negative effects on children's overall growth and an affected child may experience physical and psychological disorders (Glover, 2011, O'Hara \& Wisner, 2014; Deklava et al., 2015). Perinatal stress should therefore be addressed at the early stages of pregnancy in order to minimize its risks to the mother and baby. While a number of psychosocial interventions address prenatal well-being, often barriers (e.g., financial, childcare \& time commitments, distance and inaccessibility) prevent engagement with these programmes. Self-guided intervention programmes could potentially provide a solution, but also encounter high attrition rates. While high-intensity follow-up and engagement strategies have shown to improve retention, they are often not practical beyond the scope of research projects. Digital interventions such as smart-phone apps may facilitate stress management for pregnant women and could provide digital incentives for retention.

There is high levels of interest in using mobile apps during pregnancy and the use of pregnancy related apps has been increasing. Apps are used for accessing pregnancy-related information, tracking own health as well as bonding with their babies. Mobile apps are seen as pregnancy companions that provide psychological and emotional support (Alkhaldi et al., 2016). However, use of apps may also cause an overload of information, suggestions and guidance, leading to increased anxiety (Lupton, 2017; Tripp et al., 2014).

Pregnant women often prefer to be more vigilant about their health and well-being during the pregnancy period (Lupton, 2017). Carissoli et al (2016) studied the use of an Italian pregnancy app "BenEssere" for well-being and coping strategies for stressful situations, which received positive feedback and high levels of engagement. Another study explored the interest of perinatal women in app use and found a clear preference for apps which could be downloaded free of charge (Osma, Barrera \& Ramphos, 2016). However, engagement and usage levels are rarely explored or reported (Carissoli et al., 2016; Lupton, 2017).

We developed PositivelyPregnant, a mobile interactive app that aims to reduce distress and improve coping skills and well-being during the perinatal period (during pregnancy and the weeks after birth). This paper reports on the results of a series of initial studies that tested the effectiveness of internal prompts/guiding to retain app use.

\section{USING GUIDED PROMPTS}

The PositivelyPregnant app aims to help women manage pregnancy related challenges and transformations. It promotes positive coping strategies to alleviate the effects of stressors and offers a selection of strategies.

The app is composed of four key sections (1) Know yourself, (2) Do something, (3) Find out, and (4) Conversations. 
To explore the influence of guiding prompts on retention and app use, we incorporated notifications to encourage the user to engage with all components of the app. These guiding prompts include a series of regular smart phone notifications to the user, once a day during the first 44 days. The delivery of prompts was designed to be consistent with the recommendations of previous research (Morrison et al., 2017; Muench \& Baumel, 2017). Prompts were delivered with a controlled frequency and provided unique information each time. Research has shown that uncontrolled frequency of prompts affects the effectiveness of the prompts, which may result in ignoring or stopping the use of the app (Fry \& Neff, 2009; Morrison et al., 2017; Muench \& Baumel, 2017).

Each of these prompts is used to highlight features of the PositivelyPregnant app. In each prompt a selected app feature is described and the user is encouraged to explore its use. For example, on day 3 the user is invited to use the Breathing feature:

"Breathing: Find a quiet and comfy place, and try out Relaxation Breathing (from Audio Activities) - it takes about 8 or 9 minutes"

Similarly, on day 43 , the user is encouraged to engage with a 'Find out' topic of changing relationships, called 'Hello, Baby'. The notification contains the following text:

"If you're thinking about your baby, daydreaming, wondering - you are already building your attachment to your child. Soon, you and your child will be building a relationship together. You may hear a lot about "attachment" and "bonding". For the short story on this, and links to more, see Hello Baby."

Once a user activates the notification, they enter the app and the notification is again shown on screen as a 'guided activity' in the message board. From both the notification and from the message board the users can directly access the recommended feature. There is no obligation for users to interact with the notifications. They can ignore these and use the app as they desire.

The app also contains in-app prompts, such as the 'Tip of the Day' with positive quotes about life, pregnancy and some general tips about day-to-day life. These are only available when the user opens the app. Further prompts are incorporated directly into app features, such as mood tracking and userdefined reminders. These are only available once set up by the user.

\section{STUDY SETUP \& RESULTS}

We designed and set up a two-group experimental design study, in which participants were randomly assigned to one of two groups, a Guided Activity
Group (the experimental) and a Non-guided Activity Group (the control group). Participants in the experimental group received the guiding prompts (notifications), the participants in the control group did not receive these. The in-built (in-app) prompts and integrated prompt features were accessible to both groups.

While 88 participants started the study, at the 24week gestation period only 65 people provided feedback, and after birth only 56 people were available to be contacted. Here we report on the assessment point at 24-weeks gestation with particular focus on the effect of guiding prompts.

\subsection{App use and Satisfaction}

App use: Participants reported the following app use during the previous two weeks: two participants used it on most days at least once, eight used it a few times a week, and 30 used it once or twice in the last two weeks. 23 participants reported not using the app at all in the last two weeks, and 25 did not provide any information.

The 23 participants who reported not using the app in the last two weeks were asked why (as an open question). Eight reported having been too busy, tired or distracted by life events. Another eight participants had objections about the app: four did not find the app helpful or relevant, two felt it was boring / not user friendly, and another two questioned the benefit of using the app as they were inserting data but did not feel that they were getting anything in return. Four participants forgot to use the app - of these four participants, two were in the guided group and two in the control group. One participant had upgraded their phone and needed a new installation, another participant stated they did not want to use the app (without giving further information) and another one did not provide any information at all.

Satisfaction: Three participants were extremely satisfied, 25 were somewhat satisfied, another 25 were neither satisfied nor dissatisfied, and nine reported to be somewhat dissatisfied with the app. None of the respondents reported to be very dissatisfied and 26 provided no information.

\subsection{Suggestions and Comments}

35 participants provided suggestions to "add, remove, or change" features of the app and 23 participants provided "further comments" about the app. Both answers often contained several aspects, with general comments including feature suggestions and vice versa. We therefore summarise here the answers according to type (not according to question). Ten comments were positive in a general sense, such as "great content" (4), "helpful" (3), "easy to use" (2), and "enjoying this so far" (1). 11 concrete examples of positive 
aspects were given, such as the push notification (4), quotes in the tip of the day (4), the stressors section, and notes to self and baby, breathing, as well as reflecting on thoughts and feelings.

Ten comments mentioned including information about the baby week by week, such as "how big is the baby" or "what I can prepare?", or a count-down or diary option. Five suggestions aimed at increasing app usage through notifications or weekly updates to encourage opening the app. Two participants wished to have the app more customisable (e.g., colours, gender of the baby). A number of suggestions were for easier navigation and usability, such as adding search (2), different colours for sections (2), better contrast for text, and indicate read articles through colour change (1). Others wished to be able to take notes, export/print information, and suggested to make the app more useful for very busy people, e.g. by shortening the audio exercises/meditation shorter (2). A number of comments regarded improving selected features, such as a rating scale for feelings, or having more partner activities. One participant was looking for advice and tracking features on "more things like sleep, vomiting, water intake etc" and the ability to "talk to others". Several mentioned the lack of personalised features and likened the advice to "reading a text book" and "questionnaires".

Negative Comments: More than 30 comments were negative or explained why the app was not used. Five of these described that the participants did not experience a clear benefit from using the app or where confused by the app. 20 others felt there was too many questions, too much text, and it was too complex. A number of the women expected more modern design and better flow of the app with one writing that the app was "more like a questionnaire than a pregnancy app".

Two participants forgot to use the app, two others felt they were too busy to engage with it, and two experienced a technical problems. Four participants voiced concern about selected app content (e.g. recommendations to have a bath or to take folate vs recommendations of their midwives).

NZ Perspective: One participant expressed her satisfaction at seeing an app that acknowledged its New Zealand context. She mentioned a number of relevant points, such as acknowledgement of Maori culture (Maori are New Zealand's first nation people). She observed that other apps use American terms such as "doula" which are not in wide use in New Zealand and are therefore confusing. Another participant also explicitly welcomed the New Zealand context. As an antenatal class coordinator she has contact with many first time mums and reported that she and the first time mums she has contact with are keen on a "NZ Pregnancy App similar to The Bump but with a kiwi twist". She observed that the dominant
American apps focus on issues that are not relevant in New Zealand (such as lack of maternity cover/care) and wishes for information about LMCs (lead maternity carers) in NZ, comparable baby sizes expressed in fruit that are known in New Zealand, location-relevant foods to avoid, and information on formula and breastfeeding in New Zealand. She reported that regarding mental health, the women felt that "if they had an NZ app then a lot of anxiety or questions they have may be answered".

\subsection{Quantitative Analysis of Feedback}

Based on the participant feedback, we calculated the number of components each participant had tried. The data in Figure 1 summarises the result, showing that participants engaged with between one and 27 components.

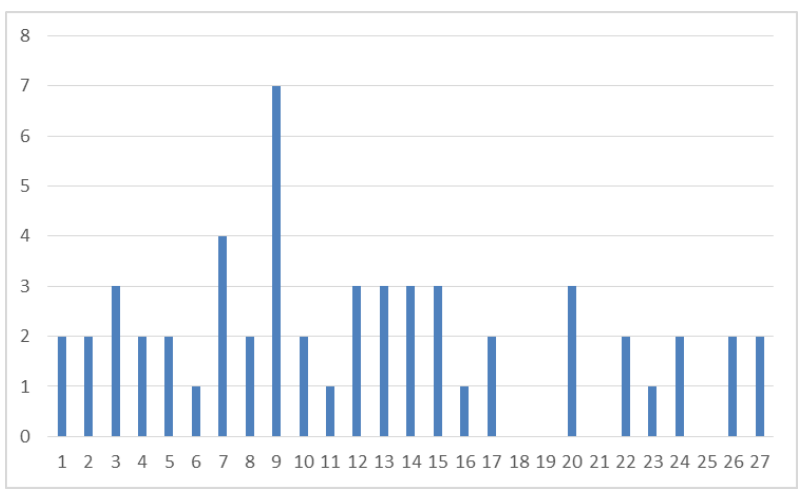

Figure 1. Number of components tried vs participants

Out of the sample of 88 participants, 63 (71.6\%) completed the 24-week gestation assessment, out of which $35(52.38 \%)$ were in the guided and 30 $(47.61 \%)$ in the control group. The Mann-Whitney U test showed no significant difference in frequency of using the PositivelyPregnant app between the two groups $(p=0.816>0.05)$. An independent samples ttest (Field, 2013) showed that there was no significant difference in number of app components used between the guided and the control group $(p=0.94, d=0.018)$. Using Mann-Whittley $U$ tests, the satisfaction levels with using the app did not differ significantly between the two groups $(p=0.195)$, and neither did the willingness to recommend to a friend $(p=0.475)$, or the extent of exploring of "Find Out" topics $(p=0.108)$. From our analysis of the user feedback, no relevant differences were observed between the two groups.

\section{DISCUSSION}

Although there is no significant difference in app use by the experimental vs the control group, a number of users who received prompts explicitly mentioned those as positive features, while a larger number of people from the control group suggested means of receiving reminders and being drawn into the app 
more regularly. It is known that participants of longterm studies have the tendency to ignore automatic triggers (prompts), which affects the frequency of app use in long-term interventions (Muench and Baumel, 2017). However, none of the participants who received prompts complained about their content or frequency. All things being equal, this still speaks clearly for the use of prompts and reminders.

One of the strengths of the study reported here and the developed app is its New Zealand context. For example, it links to numerous local online resources for appropriate organizations that are available in the participants' vicinity. The app used relevant terms and references to accessible organisations. The participant feedback on information relevant to New Zealand women was very interesting although not always directly relevant for this particular app, PositivelyPregnant. This feedback highlighted the need for a "Kiwi pregnancy app" that goes beyond the current focus of the PositivelyPregnant app on mental health. Most likely a "Kiwi pregnancy app" should be distinct from one that focusses on mental health, or at least be developed with its own context and focus, but could link to the PositivelyPregnant app.

A further strength of the study was its realistic design and execution. The app prompts and interventions in PositivelyPregnant presented a self-help program without using any external human-support such as phone or email contact. Entirely technologicallydetermined prompts and user support is the most realistic model for self-help programmes in ordinary use. Our study was able to use a true experimental design, with random assignment to experimental and control groups, and attrition from both the groups was comparable. Of the 56 people participating in a follow-up study after birth, 26 were in the control group and 29 were in the group who received regular prompts. The sample was diverse in terms of region, education, and occupation, and included women who were first time mothers as well as experienced mothers.

Shortcoming of the study are in its setup. It is known that using purely self-reported questionnaires pose threats to validity and it becomes difficult to draw firm conclusions from frequency measures (Chan, 2009 ; p.313). Out of the initial 88 participants, 26 women did not complete the 24 week assessment and it is not known whether or not they used the app. For the purpose of this study, the first follow-up assessment would have been better collected four weeks after a participant installed the app (rather than dating it by gestation). Some participants had enrolled in the study at very early stages (less than 12 weeks gestation) resulting in the most intense period of app usage being in the first few weeks which will have been a longer time before the survey at 24 weeks. Accepting women only once they were at a later point in their pregnancy would have severely limited the number of participants. A diary study in which participants record their feedback as they interact with the app would be a suitable alternative to capture earlier reactions. At the time of the survey some participants no longer remembered what was useful for them despite using a component of the app.

From the analysis of app usage data we observed that age was not associated with app usage. Andone et al. (2016) found that younger participants tend to use smartphones for longer than adults and older participants. It is possible that the relatively restricted range of ages of the childbearing women in this study constrained the possible correlation between age and app use. Ethnicity may be relevant as a study from the USA reported that African Americans and Latinos rely more on their smartphones than Caucasians for heath information, relation-related content, and job seeking (Anderson, 2015). In our study, no relevant difference could be analysed, but a larger study with a more diverse sample may shed light on this.

\section{CONCLUSION}

This paper reports on our exploration of using guided prompts in the PositivelyPregnant app. This paper provides some of the first work to investigate engagement and usage of apps for perinatal mental health. Investigation of apps for pregnant women can be found in the related work, however, engagement and usage levels are rarely explored or reported (Carissoli et al., 2016; Lupton, 2017).

Additional work on factors that might support continued use after the initial period would be helpful to the development of this and similar apps. e-Intervention is a relatively new area of research, and further exploration into persuasive practices such as incentives, tailored feedback and prompting are needed. While we found that the use of prompts had no direct influence on the satisfaction and use of the app, we recommend incorporating prompts for future users.

Addressing study design, the intervention should be tested by the participants for a medium-length fixed time period before analysis to allow insights into feedback and behaviour. User interactions need to be automatically monitored as to not rely on self-reporting.

As one of the outcomes of our study, we also identified a need for a specific New Zealand app for supporting pregnant women, in addition to the mental health app explored here. The development of this app would need to be a separate task and not a simple add-on to the PositivelyPregnant app. 


\section{REFERENCES}

Alkhaldi, G., Hamilton, F.L., Lau, R., Webster, R., Michie, S., \& Murray, E. (2016). The effectiveness of prompts to promote engagement with digital interventions: A systematic review. Journal of Medical Internet Research, 18(1): e6 Doi: http://doi.org/10.2196/jmir.4790

Anderson, M. (2015). Racial and ethnic differences in how people use mobile technology. Pew Research Centre. Retrieved from http://www.pewresearch.org/facttank/2015/04/30/racial-and-ethnicdifferences-inhow-people-use-mobile-technology/

Andone, I., Blaszkiewicz, K., Eibes, M., Trendafilov, B., Montag, C., \& Markowetz, A. (2016). How age and gender affect smartphone usage. Conference: Ubicomp'16, Researchgate. Doi: http://doi.org/10.1145/2968219.2971451

Carissoli, C., Villani, D., Triberti, S., \& Riva, G. (2016). User experience of BenEssere Mamma, a pregnancy app for women wellbeing. Annual Review of Cybertherapy and Telemedicine, 195198.

Chan, D. (2009). So why ask me? Are self-report data really that bad? In Lance, C.E. \& Vandenberg, R.J. (ed. 2010). Statistical and Methodological and Urban Legends: Doctrine, Verity and Fable in the Organizational and Social Sciences: 309-313.

Deklava, L., Lubina, K., Circenis, K., Sudraba, V., \& Millere, I. (2015). Causes of anxiety during pregnancy. Procedia- Social and Behavioural Sciences, 205: 623-626. Doi: https://doi.org/10.1016/j.sbspro.2015.09.097

Fry, J. P., \& Neff, R.A. (2009). Periodic prompts and reminders in health promotion and health behavior interventions: systematic review. Journal of Medical Internet Research,11(2): e16. Doi: http://doi.org/10.2196/jmir.1138.

Glover, V. (2011). The effects of prenatal stress on child behavioural and cognitive outcomes start at the beginning. Encyclopedia on Early Childhood Development [online]. January 2011.
Lupton, D. (2017). 'It just gives me a bit of peace of mind': Australian women's use of digital media for pregnancy and early motherhood. Societies, 7(25): 1-13. Doi: http://doi.org/10.3390/soc7030025

Morrison, L.G., Hargood, C., Pejovic, V., Geraghty, A.W.A., Lloyd, S., Goodman, N., Michaelides, D.T., Weston, A., Musolesi, M., Weal, M.J., \& Yardley, L. (2017). The effect of timing and frequency of push notifications on usage of a smartphone-based stress management intervention: An exploratory trial. PloS One, 12(1): $\quad$ e0169162. Doi: http://doi.org/10.1371/journal.pone.0169162

Muench, F., \& Baumel, A. (2017). More than a text message: Dismantling digital triggers to curate behaviour change in patient-centred health interventions. Journal of Medical Internet Research, 19(5): e147. Doi: http://doi: 10.2196/jmir.7463.

O'Hara, M.W., \& Wisner, K.L. (2014). Perinatal mental illness: Definition, description and aetiology. Best Practice and Research: Clinical Obstetrics and Gynecology, 28(1): 3-12.

Osma, J., Barrera, A.Z., \& Ramphos, E. (2016). Are pregnant and postpartum women interested in health-related apps: Implications for the prevention of perinatal depression? Cyberpsychology, Behaviour and Social Networking, 19 (6): 412-415. Doi: http://dx.doi.org/10.1089/cyber.2015.0549.

Sprenger, M., Mettler, T., \& Osma, J. (2017). Health professionals' perspective on the promotion of e-mental health apps in the context of maternal depression. PLoSOne, 12(7): e0180867 http://doi.org/10.137/journal.pone.0180867

Tripp, N., Hainey, K., Liu., A., Poulton, A., Peek, M., Kim., J., \& Nanan, R. (2014). An emerging model of maternity care: smartphone, midwife, doctor? Women Birth, 27(1): 64-7. Doi: http://doi.org/10.1016/j.wombi.2013.11.001 